\title{
Photon-flux determination by the Poisson-fitting technique with quenching corrections
}

\author{
Hiroaki Utsunomiya ${ }^{\mathrm{a}, *}$, Takafumi Watanabe ${ }^{\mathrm{a}}$, Takashi Ari-izumi ${ }^{\mathrm{a}}$, Daiki \\ Takenaka $^{\mathrm{a}}$, Takeru Araki ${ }^{\mathrm{a}}$, Kazuya Tsuji ${ }^{\mathrm{a}}$, Ioana Gheorghe ${ }^{\mathrm{b}}$, Dan M. \\ Filipescu $^{\mathrm{b}}$, Sergey Belyshev ${ }^{\mathrm{c}}$, Konstantin Stopani $^{\mathrm{c}}$, Dmytro Symochkod $^{\mathrm{d}}$, \\ Hongwei Wang ${ }^{\mathrm{e}}$, Gongtao Fan ${ }^{\mathrm{e}}$, Therese Renstrøm ${ }^{\mathrm{f}}$, Gry M. Tveten ${ }^{\mathrm{f}}$, \\ Yiu-Wing Lui ${ }^{\mathrm{g}}$, Kento Sugita ${ }^{\mathrm{h}}$, Shuji Miyamoto ${ }^{\mathrm{h}}$ \\ ${ }^{a}$ Department of Physics, Konan University, Okamoto 8-9-1, Higashinada, Kobe 658-8501, \\ Japan \\ ${ }^{b}$ ELI-NP, "Horia Hulubei" National Institute for Physics and Nuclear Engineering \\ (IFIN-HH), 30 Reactorului, O77125 Bucharest-Magurele, Romania \\ ${ }^{c}$ Lomonosov Moscow State University, Skobeltsyn Instutute of Nuclear Physics, 119991 \\ Moscow, Russia \\ ${ }^{d}$ Institut für Kernphysik, Technische Universität Darmstadt, Darmstadt, 64289, Germany \\ ${ }^{e}$ Shanghai Institute of Applied Physics, No. 2019 Jialuo Road, Jiading district, Shanghai, \\ 201800, China \\ ${ }^{f}$ Department of Physics, University of Oslo, N-0316 Oslo, Norway \\ ${ }^{g}$ Cyclotron Institute, Texas A $8 M$ University, College Station, Texas 77843, USA \\ ${ }^{h}$ Laboratory of Advanced Science and Technology for Industry, University of Hyogo, 3-1-2 \\ Kouto, Kamigori, Ako-gun, Hyogo 678-1205, Japan
}

\begin{abstract}
Single- and multi-photon spectra of pulsed $\gamma$-ray beams produced at 17,34 , and $40 \mathrm{MeV}$ in the laser Compton scattering were measured with an $8 " \times 12 "$ $\mathrm{NaI}(\mathrm{Tl})$ detector. By using the experimental single-photon spectra as the probability function of generating random numbers, response functions of the $\mathrm{NaI}(\mathrm{Tl})$ detector to $j$-fold photons $(j=2,3,4 \ldots)$ were constructed. The least-square fits to the experimental multi-photon spectra by the Poisson distribution consisting of the response functions were made. The multi-photon spectra measured at 17 and $34 \mathrm{MeV}$ follow the Poisson distribution. A quenching phenomenon of multi-photon spectra was observed for $40 \mathrm{MeV} \gamma$-rays as a result of the saturation at the photomultiplier tube of the $\mathrm{NaI}(\mathrm{Tl})$ detector. The original Poisson distributions were restored from the quenched spectra using a saturation curve
\end{abstract}

\footnotetext{
* Corresponding author

Email address: hiro@konan-u.ac.jp (Hiroaki Utsunomiya)
} 
in the form of $y=a \cdot x^{\eta}$ with $\eta=\mathrm{e}^{-b x}$. We discuss the accuracy of photon-flux determination.

Keywords: Multi-photon spectra, $\gamma$-flux determination, detector response

\section{Introduction}

The so-called pile-up method [1] or the Poisson-fitting method [2] is routinely used to monitor the photon flux in experiments with pulsed $\gamma$-ray beams produced in laser Compton scattering. The accuracy of this method of determining 5 photon flux depends on how precisely the pile-up or multi-photon spectrum follows the Poisson distribution. In this paper, we closely investigate the nature of multi-photon spectra measured for high-energy pulsed $\gamma$-ray beams and the accuracy of the photon-flux determination.

Quasi-monochromatic $\gamma$-ray beams are produced in the collision of laser photons on relativistic electrons. Since the large numbers of laser photons $\left(N_{\ell}\right)$ and electrons $\left(N_{e}\right)$ participate in the collision with a small collision probability $(p)$, pulsed $\gamma$-ray beams produced by a Q-switch laser involve multi-photons per pulse $(n)$ that are considered to follow the Poisson distribution [1, 2, 3], $P_{m}(n)$,

$$
P_{m}(n)=\frac{m^{n}}{n !} e^{-m},
$$

where $m$ is the average number of photons per $\gamma$-pulse; namely, $m=p N$ for ${ }_{10} \quad N=N_{\ell} \cdot N_{e}$.

The total number of pulsed $\gamma$-rays can be experimentally determined from multi-photon spectra measured with a $\gamma$-ray monitor detector 1 .

Experimentally, the average number of photons per $\gamma$ pulse is obtained with the pile-up method [1] by

$$
m^{e x p}=\frac{\bar{N}_{m}}{\bar{N}_{s}}
$$

where $\bar{N}_{m}$ and $\bar{N}_{s}$ are the average channel numbers of the multi- and singlephoton spectra, respectively.

The total number of $\gamma$ rays, $N_{\gamma}^{\text {tot }}$ is determined by

$$
N_{\gamma}^{\text {tot }}=m^{e x p} \times N_{\gamma}^{\text {pulse }},
$$


where $N_{\gamma}^{\text {pulse }}$ is the number of $\gamma$ pulses which is equal to the total events of multi-photon spectra, $N_{\gamma}^{\text {pulse }}=\sum_{i} N_{m}(i)$.

\section{Experiment}

Figure 1 shows the $\gamma$-ray beam line of the NewSUBARU synchrotron radiation facility. Electrons were injected at the energy of $974 \mathrm{MeV}$ from a linear accelerator into the NewSUBARU storage ring. The storage ring was operated in the top-up mode at $500 \mathrm{MHz}$ with $60 \mathrm{ps}$ electron pulse width, where the electron beam current was kept at $300.3-299.8 \mathrm{~mA}$ by automated injections of electrons from the linear accelerator whenever the current dropped below $299.8 \mathrm{~mA}$. The Talon laser $(\lambda=532 \mathrm{~nm})$ 4 was operated in the Q-switch mode 25 at $1 \mathrm{kHz}$ with $40 \mathrm{~ns}$ laser pulse width. The laser photons underwent head-on collisions with the electrons at the collision point P1 of the storage ring, where the laser pulse at $1 \mathrm{kHz}$ intersects in time with electron beam bunches at 500 $\mathrm{MHz}$ as depicted in Fig. 22. At the laser power $1 \mathrm{~W}(1 \mathrm{~J} / \mathrm{s}), N_{\ell}=2.7 \times 10^{15}$ per laser pulse. Since twenty electron bunches intersect with the laser pulse, $N_{e}$

${ }_{30}=4 \times 10^{10}$ at the electron beam current $300 \mathrm{~mA}$, resulting in $N \sim 10^{26}$. Note that a typical value of $m$ is 10 as a result of a small collision probability.

Electrons in the storage ring after the injection can be either decelerated down to $500 \mathrm{MeV}$ or accelerated up to $1460 \mathrm{MeV}$. Pulsed beams of 17 and $40 \mathrm{MeV} \gamma$-rays were produced at electron beam energies, 684 and $1061 \mathrm{MeV}$ 35 respectively, in an operation of the storage ring in the decay mode, where the electron beam current decreased with time. The electron beam energy referred to is the nominal energy which is calibrated with the accuracy of the order of $10^{-5}[\underline{5}$.

Pulsed laser-Compton scattering $\gamma$-ray beams have long been used in photoneutron cross section measurements at the experimental hutch GACKO (Gamma Collaboration Hutch of Konan University) of the NewSUBARU facility. During the photoneutron measurement, multi-photon spectra were measured with a large-volume $(8 " \times 12 ") \mathrm{NaI}(\mathrm{Tl})$ detector which has $100 \%$ detection efficiency. 
Single-photon beams were produced in a CW operation of the Talon laser with a reduced laser power. Single-photon spectra were also measured with the $\mathrm{NaI}(\mathrm{Tl})$ detector before and after the photoneutron measurement.

\section{Single-photon spectra}

\subsection{Generating single-photon spectra by random numbers}

Figure 3 shows an experimental single-photon spectrum, the number of counts $N_{s}(i)$ versus channel number $i(i=0,1,2,3, \ldots)$ measured at $34 \mathrm{MeV}$. We generated random numbers with the experimental spectrum as the probability function as follows. We define a finite numerical range of $N_{s}(i) / \sum_{i} N_{s}(i)$ $\sim N_{s}(i+1) / \sum_{i} N_{s}(i)$ and devote the range to channel $i$. We generated uniform random numbers between 0 and 1 and incremented the number of events

55 at channel $i$ when the random number falls in the range $N_{s}(i) / \sum_{i} N_{s}(i) \sim$ $N_{s}(i+1) / \sum_{i} N_{s}(i)$. A spectrum of 20,000 random numbers thus generated is shown by the solid line in Fig. 3 after normalized to the experimental spectrum. Thus, we generated random numbers with the probability function identical to the experimental single-photon spectrum.

60 3.2. Response functions to multi-photons

The $\mathrm{NaI}(\mathrm{Tl})$ detector with the time response on the order of $\mu$ s cannot resolve in time scintillation lights produced by multi-photons when they are incident on the detector during the $\gamma$-pulse width (40 ns). We can construct response functions of the $\mathrm{NaI}(\mathrm{Tl})$ detector to high-energy multi-photons by generating 65 random numbers in $j$-fold. Figure 4 shows the response functions to $j$-fold photons $(j=1,2,3, \ldots$, and 20$)$ at $34 \mathrm{MeV}$.

\section{Multi-photon spectra}

\subsection{Multi-photon spectrum at $34 \mathrm{MeV}$}

Using the response functions to $j$-fold photons, we can construct the Poisson 
to investigate how the experimental multi-photon spectrum follows the Poisson distribution. The spectrum at $34 \mathrm{MeV}$ is an ideal case for the investigation because the electron beam current, namely, the $\gamma$-ray beam intensity (the average number of photons per $\gamma$ pulse) is kept constant in the top-up operation of the storage ring.

Figure 5 shows the experimental multi-photon spectrum at $34 \mathrm{MeV}$ along with the best-fit Poisson distribution obtained in the least-squares analysis of the experimental spectrum. The experimental average number of photons per $\gamma$-pulse determined from Eq. (2) is $m^{\exp }=11.556$, while the best-fit value for the Poisson distribution is $m^{f i t}=11.545$. Thus, the average number of photons per $\gamma$-pulse for $\gamma$-ray flux can be determined with intrinsic accuracy $(0.095 \%)$ from a multi-photon spectrum that is free from time variation.

\subsection{Multi-photon spectra at $17 \mathrm{MeV}$ with time-variation}

Figure 6 shows a time-variation of the experimental multi-photon spectrum 85 at $17 \mathrm{MeV}$ in terms of the electron beam current along with the best-fit Poisson distributions. The elapsed time corresponding to each variation of the electron beam current is 5 minutes. One can see that the individual experimental spectra are again reproduced well by the Poisson distribution with different values of the average photon number. The experimental values of the average photon number obtained from Eq. (2) are listed in Table I in comparison with the bestfit values. The experimental value in principle is considered to hold the intrinsic accuracy at every moment as in the case of the $34 \mathrm{MeV}$ data. In contrast, the best-fit value represents the average photon number of the Poisson distribution integrated over the elapsed time. However, the discrepancy is still small, being less than $0.3 \%$.

\subsection{Multi-photon spectra at $40 \mathrm{MeV}$}

Finally, we discuss about a quenching phenomenon of multi-photon spectra. Figure 7 shows two multi-photon spectra at $40 \mathrm{MeV}$ at different electron beam currents, $170-155 \mathrm{~mA}$ and $245-225 \mathrm{~mA}$. The spectra were measured at an 
increased operating voltage $(500 \mathrm{~V})$ of the photo-multiplier tube (PMT) of the $\mathrm{NaI}(\mathrm{Tl})$ detector, whereas $400 \mathrm{~V}$ was applied to the PMT in the measurements at 17 and $34 \mathrm{MeV}$. One can see that the peak channel number of $j$-fold photons in the low-current spectrum (Fig. 7(a)) is progressively shifted/quenched to a low channel for $j \geq 4$ compared to that in the Poisson distribution. The solid circles in Fig. 8 show the peak channel numbers before and after saturation obtained from the low-current data. It is noted that energy non-linearity was also reported for $\operatorname{LaBr}_{3}(\mathrm{Ce})$ detectors with different PMTs [6].

The progressive shifts are most likely attributed to the saturation effect of the PMT on electron multiplication. For a large amount of scintillation light, the anode output may tend to be saturated because of the space charge effect [7. The space charge of the high electron density may suppress the current amplification in the last few dynodes as well as the charge collection efficiency between the last dynode and the anode.

The quenching more drastically manifests itself in the high-current spectrum (Fig. 7(b)) than in the low-current spectrum (Fig. reffig7(a)). The high-current spectrum is very asymmetric because of a significant quenching of the highenergy component.

We may recover the original Poisson distribution from quenching provided that a saturation curve is known. The quenching data are experimentally available up to ten-fold photons as shown in Fig. 8. However, we need a saturation curve for more than 10-fold photons when treating the high-current spectrum. We employed the form of $y=a x^{\eta}$ for the saturation curve with a unique solution $a=\eta=1$ for non-quenching and $\eta<1$ for quenching. Here $x$ and $y$ are the channel numbers before and after saturation, respectively. However, it turned out that the quenching data is not fitted when the power $\eta$ is taken to be constant. We propose a formula for $\eta$ as $\eta=(1-c) \cdot \exp (-b x)+c$ with $\eta=1$ (no quenching) at $x \rightarrow 0$ and an asymptotic value $\eta=c$ at $x \rightarrow \infty$. It is however noted that the saturation curve is not sensitive to a choice of the asymptotic value over the relevant channel range because best-fit values of $b$ are on the order of $10^{-5}$. The saturation curves for $c=0.5,0.4,0.2$, and 0 are shown in 
Fig. 8. For example, the channel number 2500 (the maximum channel number of the high-current data in Fig. 6(b)) is converted to 1453, 1457, 1465, and 1471 by the formula with $c=0,0.2,0.4$, and 0.5 , respectively. The difference which is less than $1.3 \%$ in the range of $c=0-0.5$ decreases with decreasing the channel number. Thus, we have chosen $c=0$ for simplicity.

The best-fit to the quenching data is shown by the solid curve in Fig. 8 , where $a=1.045, b=3.121 \times 10^{-5}$. Using the best-fit saturation curve, a leastsquares analysis was performed to reproduce the quenched spectra with the average number of photons of the Poisson distribution as a free parameter. The Poisson distributions recovered from quenching are also shown for the low- and high-current data in the figure. The resultant best-fit values of the average photon number for the low- and high-current data are $6.509 \pm 0.094(1.5 \%)$ and $10.789 \pm 0.266(2.5 \%)$, respectively. The uncertainty quoted represents a range of the average photon number over which the $\chi_{\nu}^{2}$ per degree of freedom varies by 1 (one standard deviation). Thus, we can determine the average number of photons per $\gamma$-pulse even from quenched spectra with high accuracy.

\section{Conclusions}

Exact determination of $\gamma$-flux is crucial for the experiment aimed to accurately measure the cross section of photonuclear reactions. Experimental multi-photon spectra measured for pulsed beams of the laser-Compton scattering $\gamma$-rays follow the Poisson distribution. Quenched multi-photon spectra were observed for $40 \mathrm{MeV} \gamma$-rays as a saturation phenomenon of a photomultiplier tube. It was shown that the original Poisson distribution is restored from the quenched spectra using the saturation curve proposed in the present paper.

The $\gamma$-ray flux is determined from multi-photon spectra without quenching with the intrinsic accuracy less than $0.1 \%$ and from those with quenching with the accuracy $\leq 2.5 \%$ unless multi-photon events are partly lost due to saturation. 


\section{Acknowledgements}

This work was supported by the IAEA under the contract numbers for the CRP-F41032, JPN-20564, ROM-20476, and RUS-20501 and by the Premier Project of the Konan University in the category of Research and Education. I.G. and D.F. acknowledge the support from the Extreme Light Infrastructure Nuclear Physics (ELI-NP) Phase II, a project co-financed by the Romanian Government and the European Union through the European Regional Development Fund - the Competitiveness Operational Programme (1/07.07.2016, COP,

ID 1334). D.S. acknowledges the support of Deutsche Forschungsgemeinschaft through grant No. SFB 1245. G.M.T acknowledges funding from the Research Council of Norway under grant no. 262952/F20. 


\section{References}

170 [1] T. Kondo et al., Determination of the number of pulsed laser-Compton scattering photons, Nucl. Instrum. Meth. A 659 (2011) 462.

[2] H. Toyokawa et al., Flux Measurement of the Laser-Compton-Backscattered Photons with a Poisson Fitting Method, IEEE Trans. Nucl. Sci. 47 (2000) 1954.

[3] T. Kii et al., Photon Counting Method for High Flux Pulsed Laser-Compton Backscattered Photons Based on Poisson model, in Proceedings of the 12th Symposium on Accelerator Science and Technology, ed. Yasushige Yano (The Institute of Physical and Chemical Research (RIKEN), Wako, Japan (1999) 484 .

[4] http://www.spectra-physics.com/products/q-switched-lasers/talon.

[5] H. Utsunomiya et al., Energy Calibration of the NewSUBARU Storage Ring for Laser Compton-Scattering Gamma Rays and Applications, IEEE Trans. Nucl. Sci. 61 (2014) 1252.

[6] G. Gosta et al., Response function and linearity for high energy $\gamma$-rays in large volume LaBr3:Ce detectors, Nucl. Instrum. Meth. A 879 (2018) 92.

[7] T. Hakamata et al., Editorial Committee, Photomultiplier Tubes - Basics and Applications, HAMAMATSU PHOTONICS K. K. Electron Tube Division Third Edition (Edition 3a with minor revisions) (2007) 90. 
Table 1: The experimental and best-fit values of the average number of photons

\begin{tabular}{lccc}
\hline \hline $\begin{array}{l}\gamma \text { energy } \\
(\mathrm{MeV})\end{array}$ & $\begin{array}{c}\text { Electron beam current } \\
(\mathrm{mA})\end{array}$ & Experimental value & Best-fit value \\
\hline 34 & $300(300.3-299.8)$ & 11.556 & 11.545 \\
17 & $248-240$ & 13.072 & 13.029 \\
& $233-226$ & 11.635 & 11.609 \\
& $212-206$ & 10.06 & 10.184 \\
& $171-166$ & 6.952 & 6.948 \\
& $170-155$ & & $6.509^{*}$ \\
40 & $245-225$ & & $10.789^{*}$ \\
\hline \hline
\end{tabular}

* The average photon number of the restored Poisson distribution 


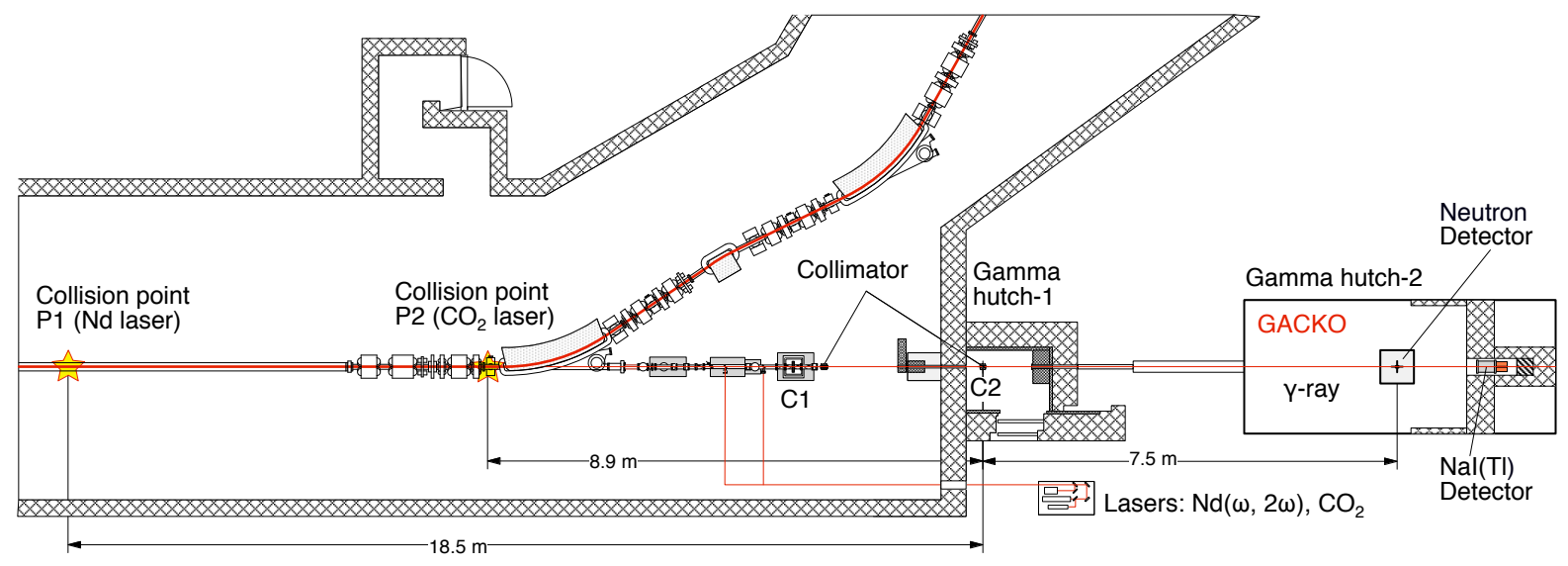

Figure 1: (Color online) The $\gamma$-ray beam line and the experimental hutch GACKO of the NewSUBARU synchrotron radiation facility. 


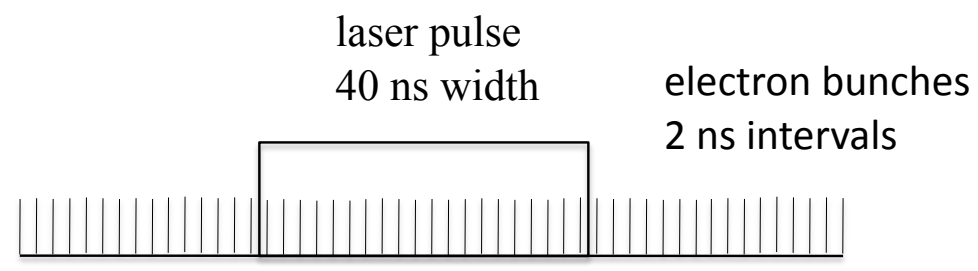

Figure 2: Collisions between laser photos and relativistic electrons. 


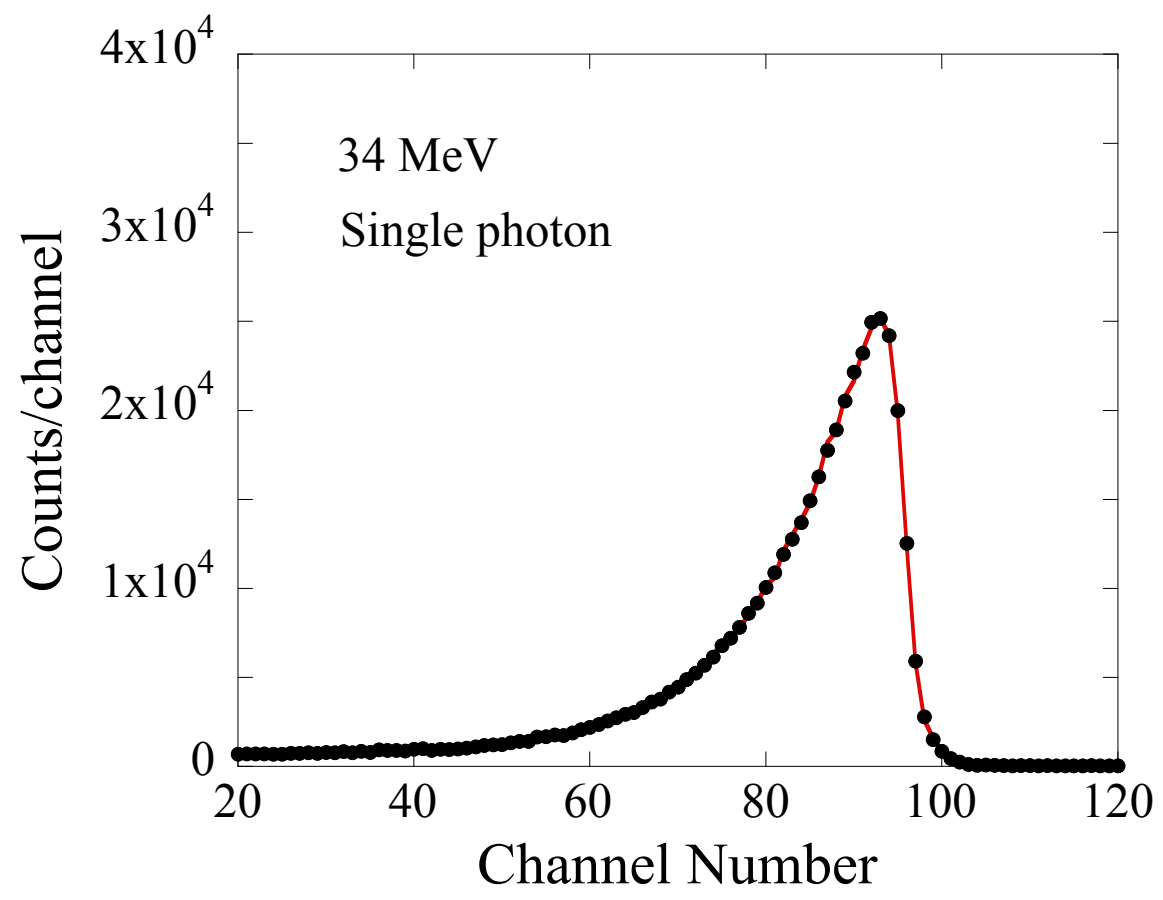

Figure 3: (Color online) Single-photon spectrum at $34 \mathrm{MeV}$. The solid line shows a spectrum of 200,000 random numbers generated with the experimental single photon spectrum as the probability function. The spectrum of random numbers are normalized to the experimental spectrum. 


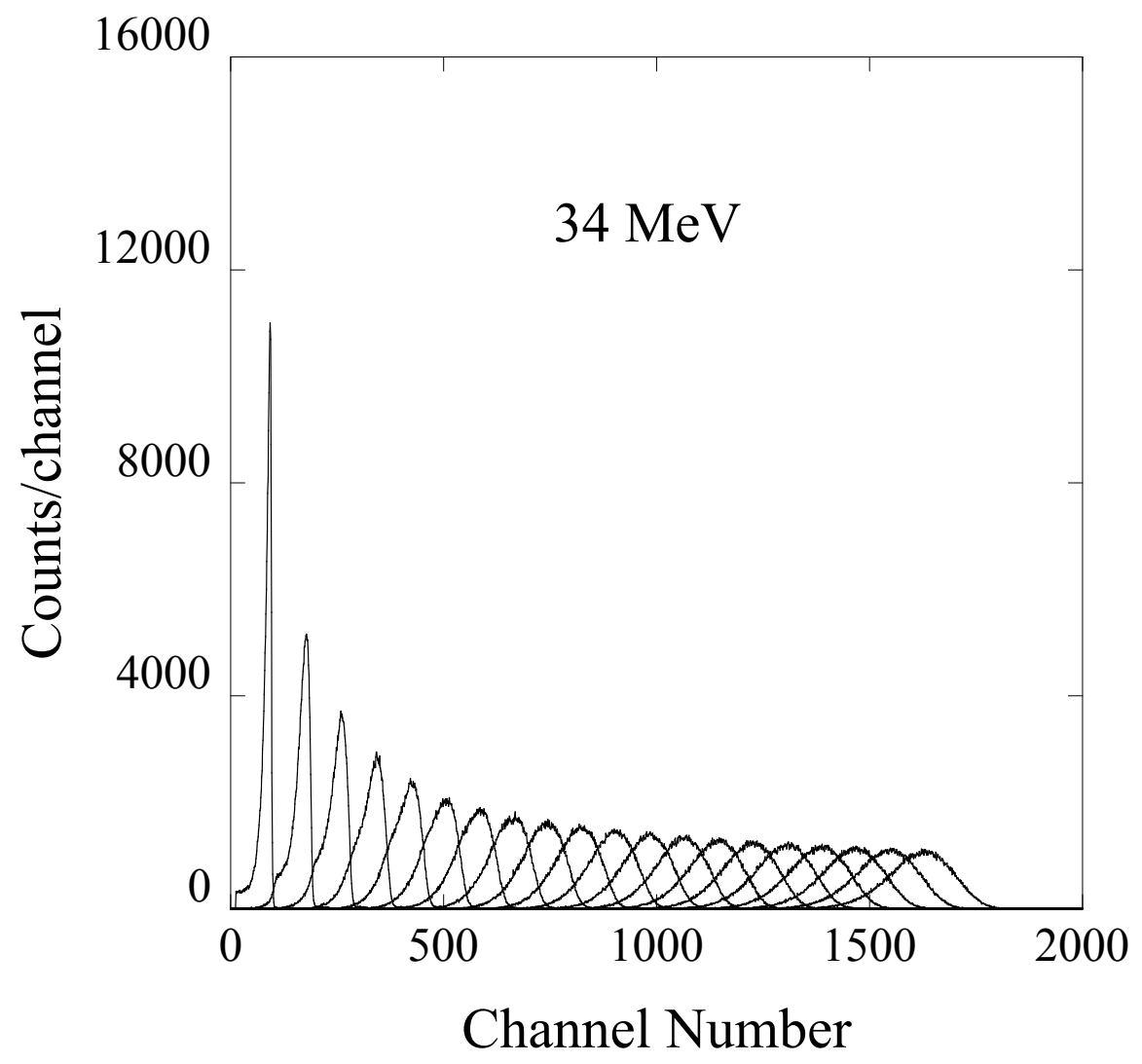

Figure 4: Response functions to $j$-fold photons $(j=1,2,3, \ldots, 20)$ at $34 \mathrm{MeV}$. 


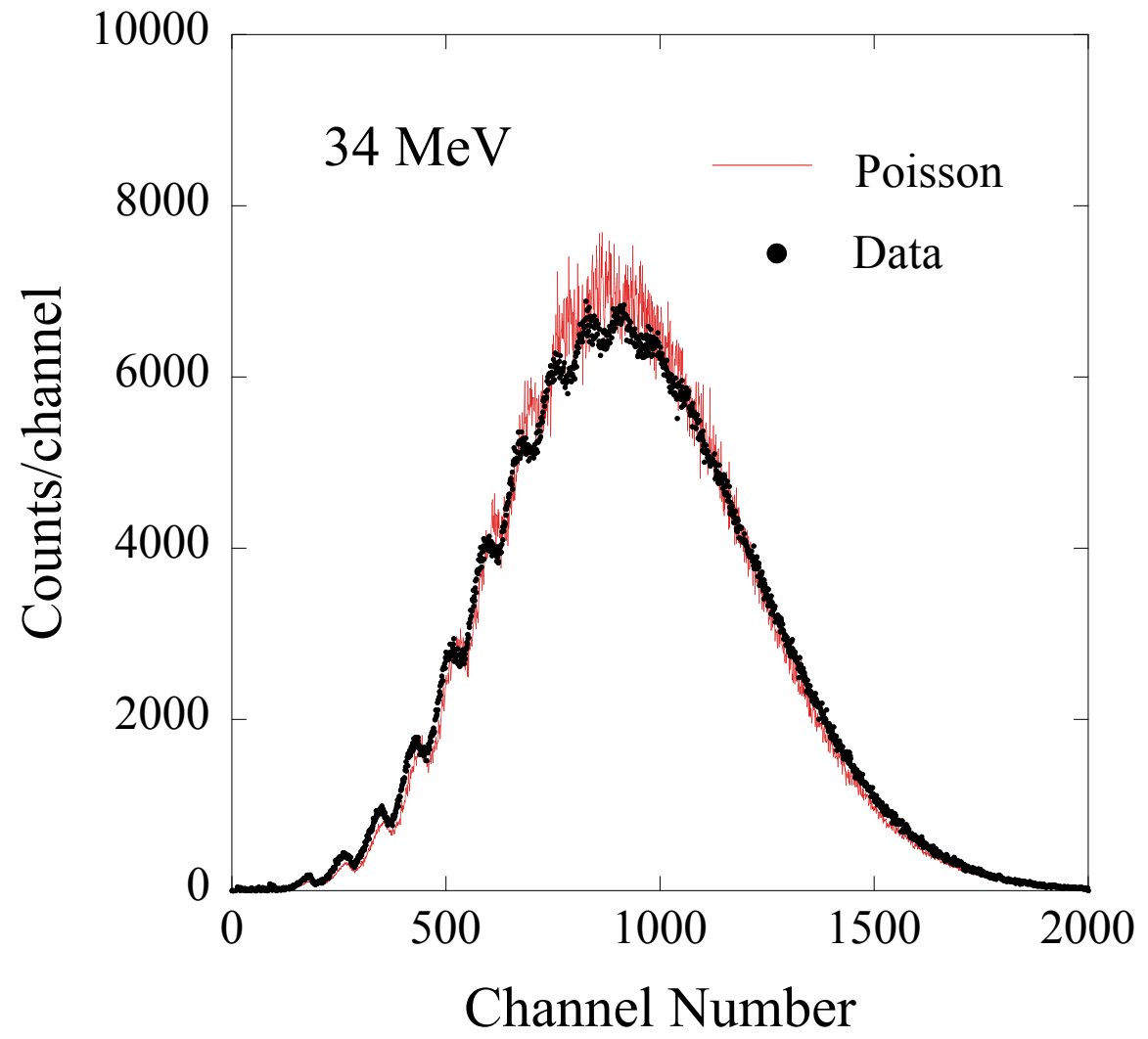

Figure 5: (Color online) Experimental multi-photon spectrum at $34 \mathrm{MeV}$ in comparison with the best-fit Poisson distribution. 


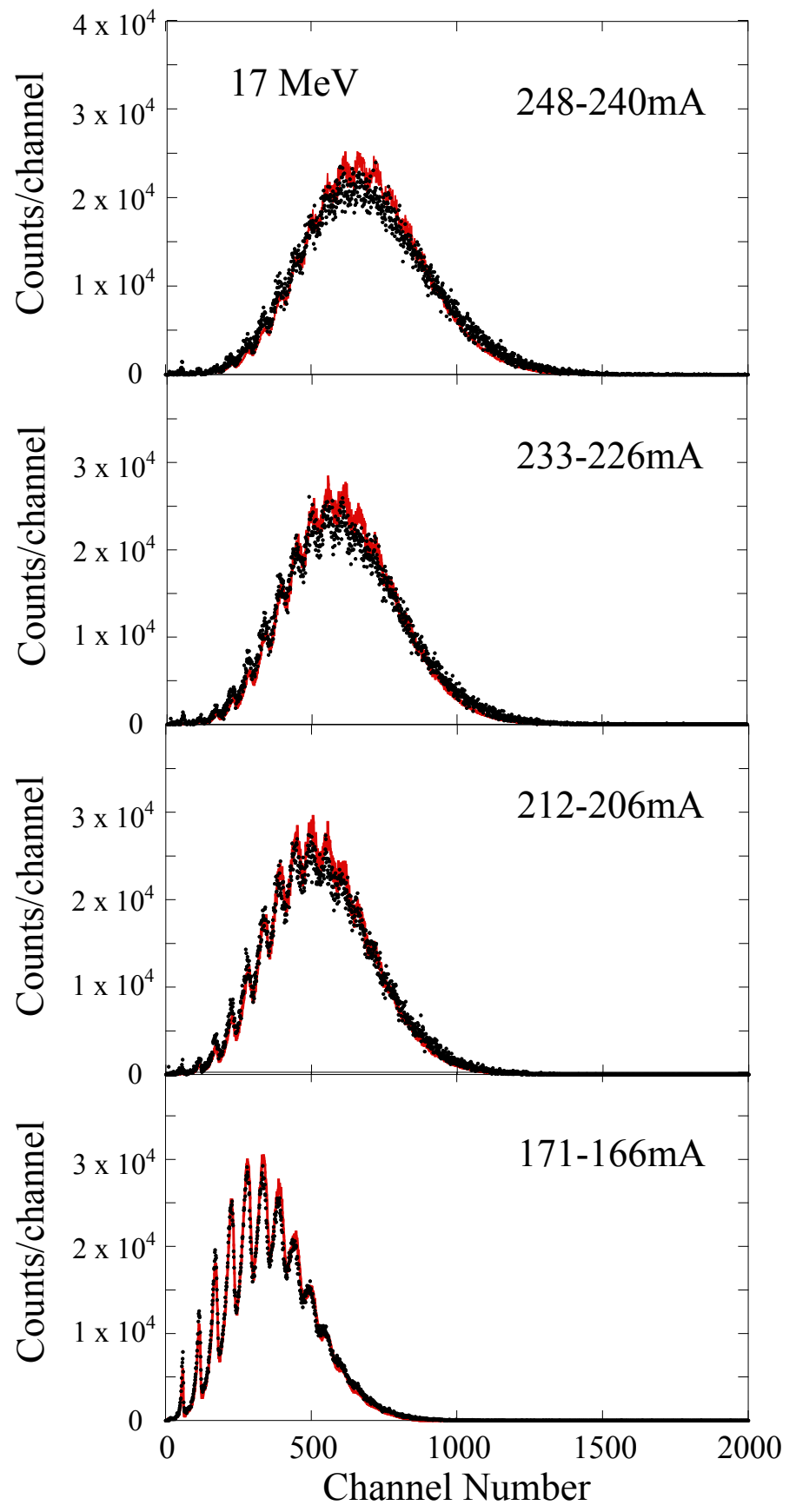

Figure 6: (Color online) Experimental multi-photon spectra with time-variation at $17 \mathrm{MeV}$ in comparison with the best-fit Poisson distributions. 

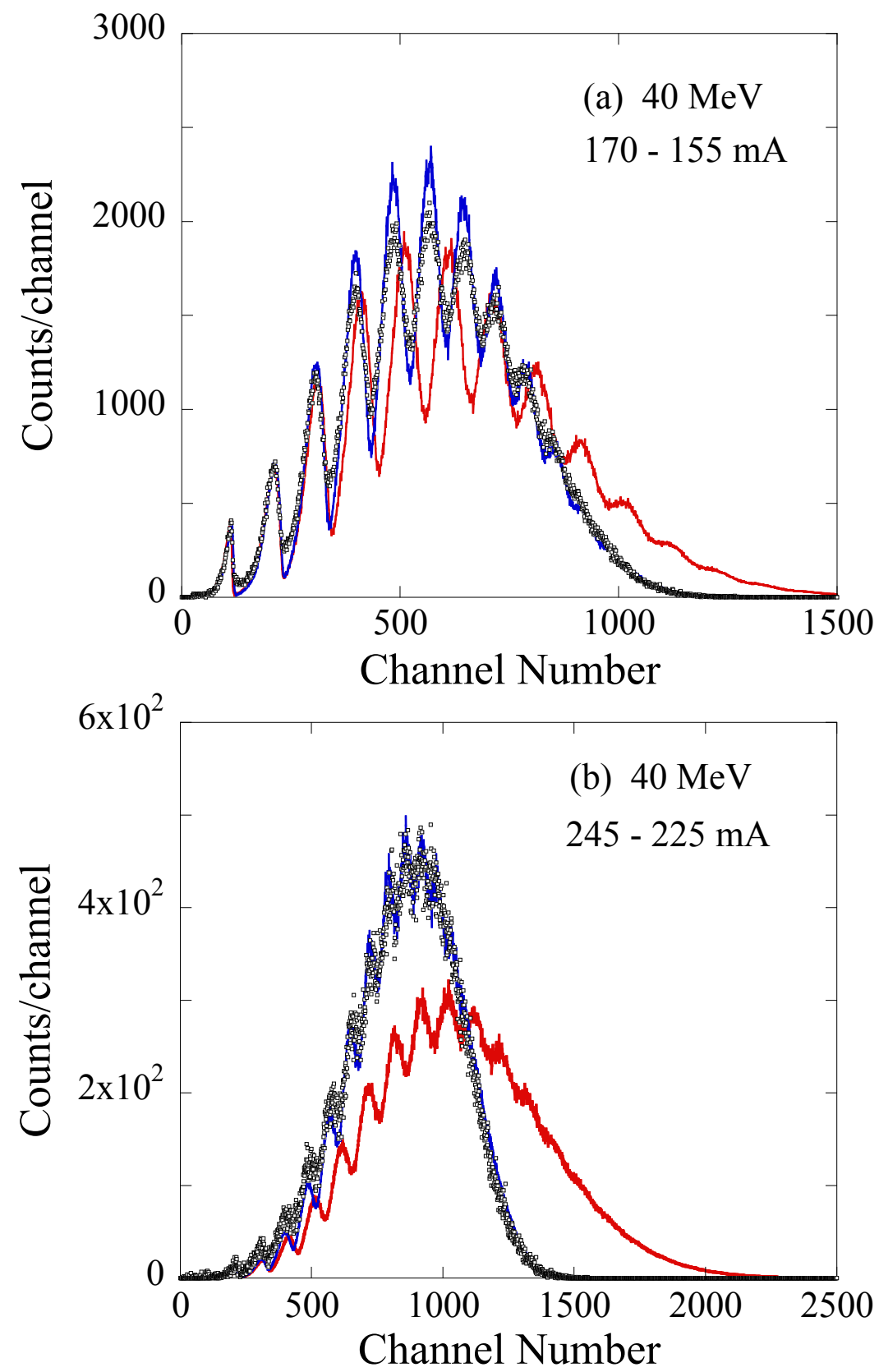

Figure 7: (Color online) Experimental multi-photon spectra at $40 \mathrm{MeV}$ : (a) low-current data; (b) high-current data. Both the original and quenched Poisson distributions obtained in the least-squares analysis are shown by the solid lines. 


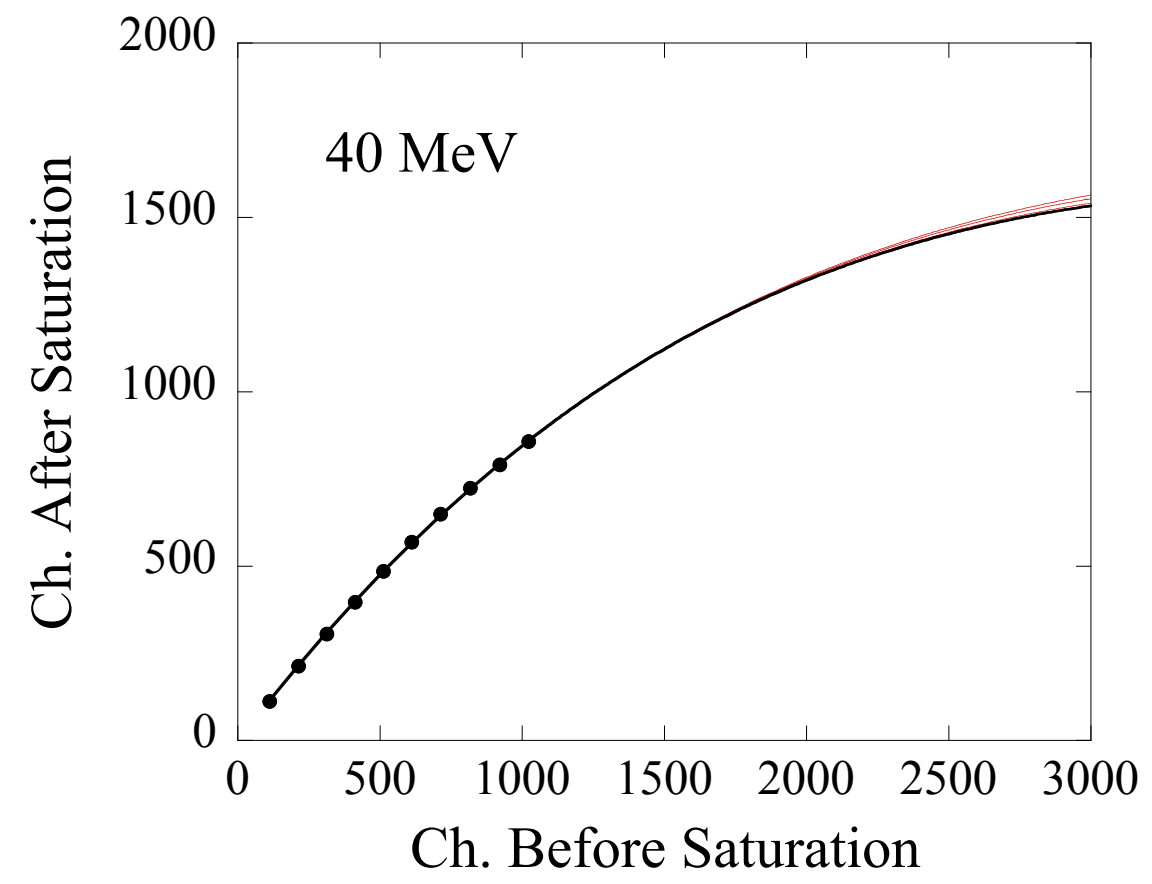

Figure 8: (Color online) Quenching data of the relation between the channel numbers before and after the quenching at $40 \mathrm{MeV}$. The saturation curve is also shown by the solid line. See text for details. 